\title{
THE SIGNIFICANCE OF BURIED WEED SEEDS IN AGRICULTURE
}

\author{
By G. S. HARRIS, Grasslands Division, Department of Scientific \\ and Industrial Research, Palmerston North
}

The question "where do all the weeds come from?" is one which has plagued the gardener and intrigued the botanist and agriculturalist for many centuries. Often plants which are not common in the area literally appear to have "come from nowhere".

Seed blown in by the wind (thistle seeds are an example) are of fairly obvious origin and are readily accepted as a source of weed plants; seeds carried by animals (barley grass (Hordeum murinum) in the fleece or clover seed in the dung) are perhaps not so obvious, as are seeds such as nightshade (Solanum nigrum) transported long distances by birds. Really puzzling are those appearing where there is no easily recognised seed source.

Although there is occasional mention in the literature as far back as the Greek philosophers (1) of the fact that some seeds keep their vitality better than others, it was not until about 1832 that much attention was paid to the possibility of certain seeds remaining viable in the soil over extended periods of time. Between 1832 and the commencement of the present century much was written on the subject, but with few exceptions the accounts were based on general observations and speculation rather than on experiment.

With Duvel (2), in 1904, experimental investigation began, and today the emphasis has naturally shifted from the general to the particular.

Work to date has shown that the life of seeds, as of plants, varies greatly with the different families, genera, and species, but that there is no relation between the longevity of the plants themselves and the viable life of the seeds they bear. Certain willow (Salix spp.) and poplar (Populus spp.)

trees, with a lifetime measured in tens of years, bear seeds which remain viable for a few days only; some annual herbs, with a lifetime of a few months, produce seeds whose lifetime may be a decade. Provided a seed has been thoroughly matured, its life period, within its own particular time range, is largely dependent on its environment, and loss of vitality is especially marked in seeds stored in places of high temperatures and humidities.

Strangely enough, there are comparatively few cultivated plants whose seeds retain their viability for any length of time when buried in the soil, but the reverse is true in many cases with plants we have come to regard as weeds. Many seeds are better preserved the deeper they are buried, for at depth below the surface the soil moisture is more even, oxygen supply is lowered, and temperature reduced and less prone to fluctuation. Since most seeds, for germination, need adequate moisture, a good oxygen supply, and in many cases daily alternation of temperature, such preservation by deep burial is not surprising; and, equally, seeds brought up from such depths, provided they are still viable, frequently respond to surface conditions by prompt germination.

In any study of buried seeds it is imperative to pay some attention to the factors responsible for the persistence of viable seeds, and the phenomenon known as dormancy is one of the more important of these. Definition of dormant as "inactive; in a state of suspended animation" sums up the situation very well, as does the alternative of "seeds which, though viable, are temporarily incapable of germinating under conditions normally conducive to germination". Temporarily, in the latter case, may imply a considerable time.

The more common type of dormancy is that associated with what is known as "after ripening", and concerns the period from the time the 
seed reaches maturity to the time when, under favourable conditions, it germinates. This dormancy is very widespread among plants, and may cover such a short period that its presence is easily overlooked, or may persist for much longer. Its advantage to the plant is undoubtedly its "survival value", if such a term may be used. Seed shed when mature, and all germinating at once, renders this seedling crop vulnerable to local flood or drought, or to other hazards. A whole generation may be wiped out at one stroke and the plant population decimated. A dormant period following seed fall, however, ensures a spread of germination, and provides an insurance for the species against its wholesale destruction. Fathen (Chenopodium spp.) and docks (Rumex spp.) are weeds where spread of germination will be familiar.

The second type of dormancy which may occur is one in which, for various rather obscure reasons, seed does not germinate at once when brought into conditions which are normally favourable. In spite of considerable work on this aspect, not a great deal is known of the biological changes which are responsible for the initiation of the condition or for its disappearance. Of the mechanical factors concerned, work has been done on valve mechanisms (Hyde, 3 ) in those seeds where a hard seed coat presents a barrier impermeable to the ingress of moisture. Dormancy of this type may persist for quite long periods; five to 50 years is by no means unusual, depending on the species.

The practical farming importance of dormancy, so far as weed seeds are concerned, was well summed up by the late E. O. C. Hyde in an address last year (4) when he said: "The time over which germination is spread is an inherent characteristic of the species though subject to modification by the environment. With some species it is a short time, a few weeks or months. With others it is years or decades. Here we have an important difference. On the one hand we have a reckless enemy who throws all his troops into the first battle. On the other hand we have a cautious enemy who holds most of his troops in reserve, preferring a war of attrition. He may continue to skirmish for 50 years, always ready to exploit opportunity. It is essential to know which enemy we are up against so that the right strategy may be adopted."

How, then, does one go about finding out what seeds lie buried in the soil, how many there are and of what kind, and, more important, whether they are capable of germination? The mechanics of the process, though somewhat tedious, are comparatively simple. The evaluation of the results may be a little more difficult.

Samples of soil are taken with an ordinary soil sampler. This gives cores of soil of a known surface area, and depth can be adjusted as desired, depending on soil conditions, to $12 \mathrm{in}$. or more. Our work to date has been a survey of buried seed populations; the usual number of cores taken has been 50 to 100 for each area. This has proved sufficient to indicate the presence or absence of a particular species, though probably insufficient for detailed paddock to paddock comparisons.

The cores from each area are bulked and brought to the laboratory with as little delay as possible. The first step is to get rid of the surplus inorganic soil material, and this is done by placing the cores in a bag of very fine-mesh nylon material and gently kneading the contents in a copious flow of water. Soil aggregates are thus broken down, and clay and fine silt washed out through the mesh, though this is sufficiently fine to retain such small seeds as toadrush (Juncus bufonius) and browntop (Agrostis tenuis).

The much diminished bulk of material is then transferred to graded sieves and washed through with gentle shaking and a copious flow of water. The result is a series of subsamples which contain the larger soil particles such as gravel and small stones, vegetable debris, and the seed content. Where one type of seed only is of interest, the washing can be speeded up by choice of an appropriate sieve to retain the desired seed and let the rest of the material through. 
The washed material is now dried at a moderate temperature ( 30 degrees to 40 degrees) in a current of air. The whole process from sampling to drying of sieved material is done quickly to avoid as far as possible any germination of seeds in the sample under the improved moisture, oxygen, and temperature conditions once the cores are out of the soil. Once dried, however, the samples may be stored for several weeks, if necessary, before further processing.

As has been mentioned the sample is now a mixture of inorganic soil particles and organic plant material, and advantage is taken of density differences to make the necessary separation. The sample is stirred into a quantity of carbon tetrachloride in a large beaker with the result that the organic material including seed floats and can be decanted off, while the inorganic fraction sinks to the bottom. Carbon tetrachloride was chosen, after considerable trial of other materials, for several reasons. It is of the correct density to give ready separation (except, unfortunately, of pumice in some samples), it is fairly readily available at a reasonable cost, it evaporates readily from the treated sample in a current of warm air, and short immersion in it does not affect the germination of viable seeds. A disadvantage is the danger from fumes which necessitates adequate ventilation when handling it.

The vegetable material is filtered through glass wool and then spread on absorbent paper in a warm place with adequate ventilation. Drying occurs rapidly and most of the plant debris can then be removed by gentle rubbing and winnowing. Passage through a series of small graded sieves effects considerable seed separation by size, and the final step is hand sorting, if necessary under a dissecting microscope. Viable seeds may be determined by any of the standard germination tests.

The range of species present in typical samples, and an indication of numbers, are given in Table 1 . These results are typical of those obtained from pasture or cultivated areas in the North Island.

While the figures in the table are impressive enough, they by no means represent the maximum numbers encountered. In one sample, from a fiverepresent the maximum numbers encountered. In one sample, from per acre, 16 million dock, and 160 million others. Under such conditions the probability of weed competition with any succeeding crop, arable or pastoral, is self-evident, as is the cost of adequate weed control measures.

Seeds are not distributed at random through the soil to an indefinite depth. A study of distribution, inch by inch, downward, revealed three classes of seeds. Firstly, there are what might be termed "ephemeral" seeds confined to the top inch of soil, such species as spear thistle (Cirsium lanceolatum) and goosegrass (Bromus mollis). Then there are those seeds which, while most abundant in the top inch, also occur at greater depths, such species as plantain (Plantago spp.) and Poa annua, which are pasture weeds with persistent seeds. And, thirdly, there are seeds less abundant in the top layers than deeper down. These latter species are usually weeds of cultivated land, and presumably the smaller population in the top inch of soil is due to the more favourable conditions there having acted to shorten the dormant period with the resultant germination reducing the seed population. Dock and twincress (Coronopus didymus) are examples of this group.

Such distributions of buried seed would suggest various possible methods of control of undesirable species. The first consideration could well be that of maintaining, in areas under pasture, a dense cover of grasses and clover which by its smothering and competitive effect will deter germination and establishment of seedlings from seed in the top inch of soil. The inhibitory effect on germination of urine patches under heavy stocking can also be important. Such an ideal is well worth aiming for, but it must be admitted important. Such an ideal is well worth aiming for, but it must be admitted less than desired. On arable land, of course, such a cover is virtually impossible, but often topping with a mower can do much to prevent seeding of weeds. 


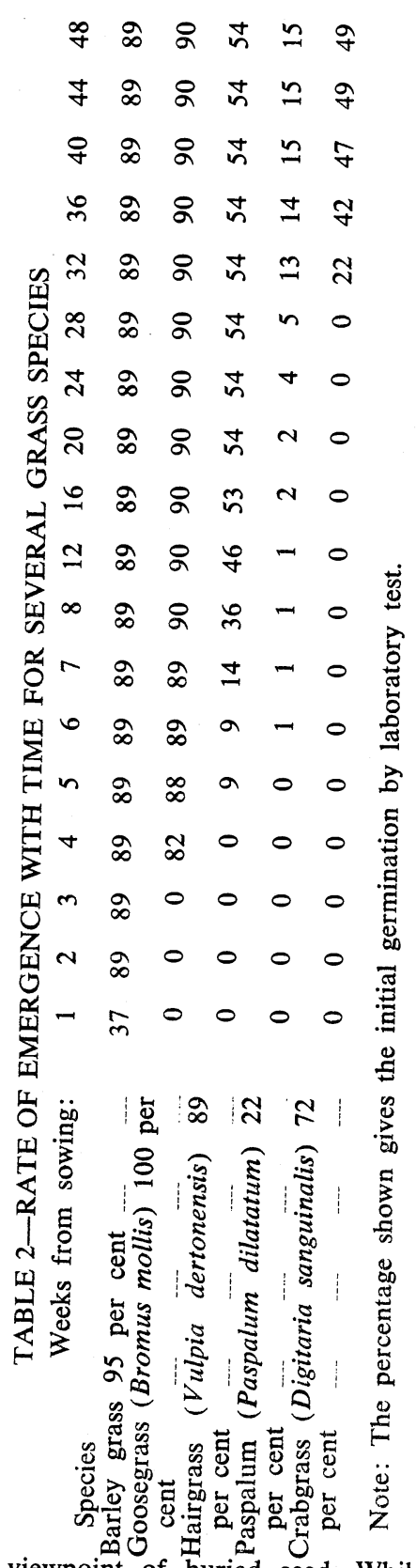

Should the seed be chiefly in the top inch of soil, there are possibilities in a really deep ploughing to completely bury the top layers of soil and bring up clean soil from lower down. The danger of bringing up from deeper layers such long-lived seeds as docks should not be forgotten, nor should the possibility that, if not turned in deep enough, subsequent cultivations may return to the surface the original weedy topsoil and thus perpetuate the problem. On certain soils the deep ploughing itself may not easily be feasible.

It may even be more profitable to cultivate several times to a shallow depth to encourage seeds near the top to germinate, and then deal with them by mechanical or chemical means until the seed supply is depleted.

Another possibility would be to apply to the surface soil some chemical which, in its passage down through the various layers, would kill all seeds which lay in its course. "Naturally, there are several "ifs" and "buts" in such a procedure. First, a substance with the required properties which will not only kill the seeds but leave the soil fit for early sowing of a crop; second, something capable of deep penetration in the soil at a sufficient potency to retain its seed-killing properties; and, third, having arrived there something capable of penetrating the formidable seed-coat barriers present in some of our worst weed seeds.

There is another angle to such "killall" weed treatments which bears consideration. There is present in the soil seed of valuable pasture clovers and quite frequently, grasses. This is useful in providing a reservoir for times of emergency (5) when the pasture above is depleted by flood or drought. This buried seed should not be unnecessarily reduced. That dormant clover seeds may constitute a problem when a pasture area is used for certified seed production is a specific case calling for the elimination of all buried seed.

So much for generalities. There is one weed problem which has become, of recent years, an increasingly important one in many parts of New Zealand, and that is the spread of barley grass. We at Grasslands are particularly interested, for besides being a grass it is a pasture weed, and some investigation has been made of its occurrence and spread from the viewpoint of buried seed. While much is still to be learnt, and several incidental puzzles have arisen, what has been found out is worthy of being placed on record. 


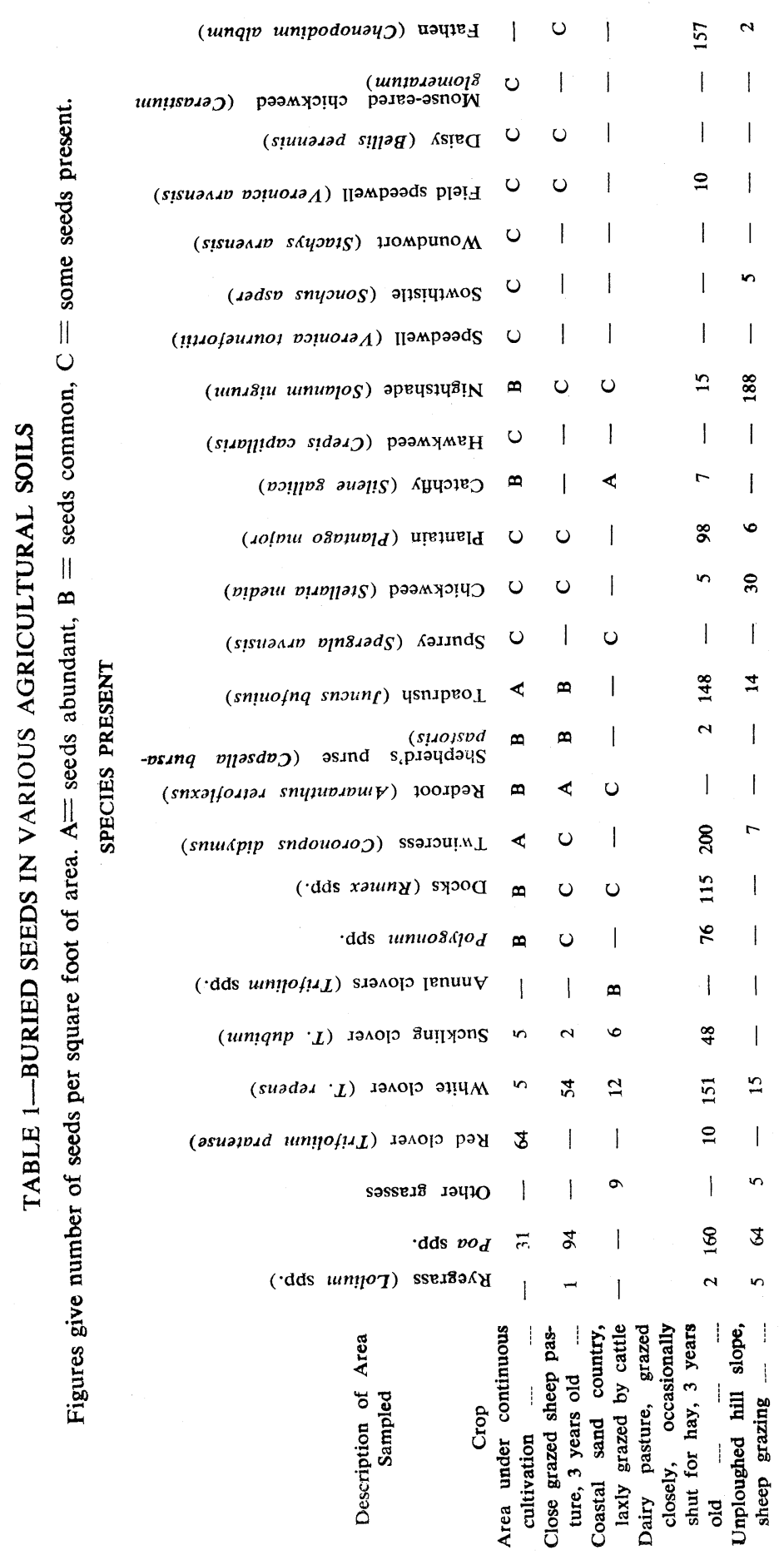

89 
In the course of a study of seed dormancy in a wide range of grass species, barley grass first drew attention because of the rapidity and completeness with which it germinated. All the grass seeds used in the investigation were harvested, when ripe, from plants in the field, and were sown out in beds of sterilised soil under field conditions. Counts of new seedlings were made each week and plants were removed as soon as they were large enough for positive identification. Typical figures showing the numbers in Table 2 .

The grasses shown in the table do not cover the full range of species studied ( 26 in all), but illustrate the rapidity of field germination, or degrees of dormancy, which were encountered. The striking features shown in the table are that, with barley grass, germination reached 37 per cent in one week from sowing, 89 per cent in two weeks, and in a period of 15 months since sowing not a single further seedling has appeared, whereas crabgrass (Digitaria sanguinalis) did not reach maximum germination until 40 weeks had elapsed.

This indicates that with barley grass the after-ripening period is practically nil, and that prolonged dormancy is absent. On the other hand there is no doubt that we do see a spring crop of barley grass seedlings in the field.

While the seed used in these trials had been collected in the field when ripe and sown at once, as mentioned earlier, there seemed a possibility that seed falling naturally on the soil might perhaps behave differently. This could best be decided by a field sampling, and during the past year such an investigation, as part of a more extensive survey, has been carried out.

Samples from areas where barley grass was known to have seeded profusely were taken first in July 1958, then in November, and again in March 1959. Any seed found in the July sampling would be dormant seeds from the previous season. Should dormancy last only from late summer through the winter to the next spring, then any dormant seeds found in July should have disappeared by November. If seeds were still present, then they carried over, not for one season only, but from one year to the next. Finally, sampling in the following autumn would give an indication of the build-up of the new season's crop of seed. The results of the field samplings are shown in Table 3.

\section{TABLE 3-DORMANT BARLEY GRASS SEEDS AT SUCCESSIVE SAMPLINGS}

Figures for number of seeds per 50 cores, 4 in. deep

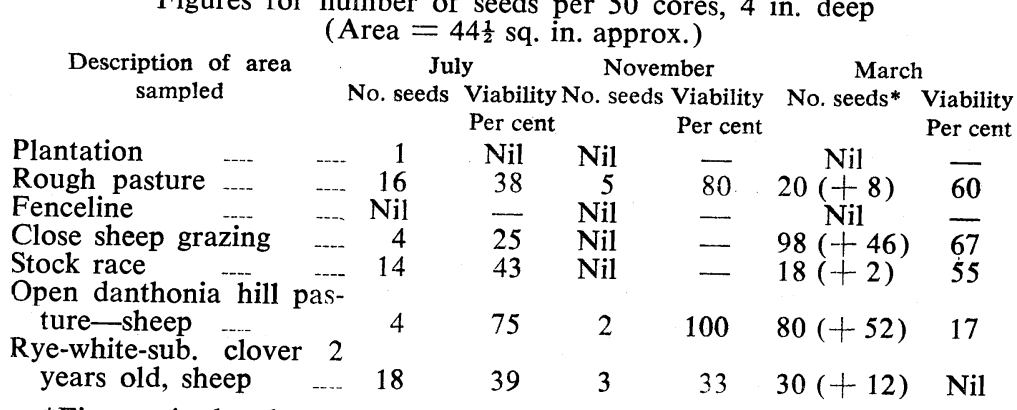

*Figures in brackets (March sampling) show numbers of seeds which had already germinated at time of sampling.

The figures for the July sampling showed appreciable numbers of seeds present at that time, and that about 40 per cent were viable. Compared with the high initial viability of fresh seed ( 90 per cent or better) it is apparent that a rapid decline had occurred in those seeds which remained in the soil over the winter period. 
The November sampling indicated that practically all the seeds remaining after July had either died and decomposed, or had germinated. The few seeds found had a high germination, and are reasonably explained as new season's seed, since early seed heads containing plump seeds were present when the areas were sampled.

A good indication of the increase in seed numbers from a single seeding season can be obtained from the March figures. The high proportions of germinated seeds are a reflection of the effect of rain about two weeks before sampling, following an unusually dry period. These seeds, obviously viable, when added to the proportion of dormant seeds proved viable by test, show that the build-up is from new season's seed, confirming that there is virtually no carry-over of seed from year to year.

It was noteworthy that in all samplings the seeds found were entangled in the surface vegetation of the cores, or in the plant roots in the top $\frac{1}{2}$ in of soil. In no case were seeds observed more than 1 in. down with the exception of one area where a closely allied species (Hordeum marinum) was present on a peat soil. Here the soil showed polygonal shrinkage cracks on drying out in the summer, offering opportunity for deep burial where seeds fell into the cracks in the ground.

If we take four plants per square foot as a figure for a fairly dense stand of barley grass and accept that each plant produces 10 seed heads each containing 50 viable seeds (and these are all conservative figures), we have a potential 2,000 seedlings per square foot. The highest figure in the March sampling would indicate a possible 350 seedlings per square foot of area from shed seed including those which may remain dormant until spring. When all seed and seedling hazards are considered this figure is surprisingly large, and can easily account for the dense stands of barley grass which are frequently met with.

Finally a brief mention of the factors involved in barley grass spread which have actually been met with during the survey. Animals, particularly sheep, carry large numbers of seeds; farm implements travelling across seeding barley grass transport seeds on tyres and tracks; on open hill slopes wind rolls the broken-off seed heads over surprising distances; hay sold from areas where barley grass is prevalent can be responsible for transfer from one district to another; seed cleanings sown to provide cover to prevent erosion on a light fluffy soil contained barley grass; and trouser cuffs provide an excellent means of dispersal

To sum up buried viable barley grass seeds in significant numbers do occur. They may last over one winter, but certainly no longer. Unless seeding is prevented, there can be a rapid and appreciable build-up in buried seed numbers by the end of the summer, but this will not last for long owing to prompt germination of the majority of the shed seeds. It is doubtful whether buried is an apt term with reference to barley grass, since under normal circumstances the seeds found in sampling were on, or within $\frac{1}{2}$ in. of, the surface.

\section{ACKNOWLEDGMENT}

Acknowledgment is made of the technical assistance of Miss M. A. McLeavey.

\section{REFERENCES}

1. Turner, J. H. Kew Bull, Misc. Inf. No. 6. 1933

2. Duvel, J. W. T. U.S.D.A. Bur. P1. Ind. Bull. 1 viii. 1904

3. Hyde, E. O. C. Ann. Bot. N.S. XVIII, 241-256. 195

5. Hyde, E. O. C., and Suckling, F. E. T. N.Z.J. Sci. Tech. A34: 375-85. 1953.

\section{DISCUSSION}

Q.- - Has $\mathrm{Mr}$ Harris carried out investigations on the viability of ragwort seed?

A.-We are conducting experiments at present and will know in two to 
three years' time. It appears so far that the seed will last a year or two but not much longer, as much seed germinates quickly.

Q.-In the Tauranga district the spread of Bathurst burr is causing trouble. I have seen deep drains dug several feet deep and Bathurst burr germinate soon after from quite deep layers apparently from buried seed. Has any work been done on the longevity of Bathurst burr seed?

A. - I have had no personal experience with Bathurst burr, but I think you must consider that some of the seed that germinated in the drains could have been carried there by the mechanical equipment used in digging the drains.

Q. - If a paddock is cut in spring with barley grass seed heads in the immature stage, will the seed germinate?

A.-Provided the seed heads have emerged from the sheath or boot, the seed probably will be viable. A tetrazolium test of seed from immature seed heads gave 56 per cent germination in 24 hours.

Q.-What about using chemicals that prevent the formation of viable seed?

A.-If such chemicals could be produced, they should be very useful, probably in conjunction with spraying with dalapon at other periods to eradicate barley grass.

Q.-Would Mr Harris comment on my experience with paspalum germination? If the seed is sown in November, an immediate strike is obtained. If it is sown in March, none germinates and none the following spring, but a strike the spring after.

A.-This sounds similar to Digitaria where there is often no immediate germination and then after a long period a burst of germination. With seed planted in early summer quite a good immediate strike was obtained. In my reported trial the seed was set out later and there was no germination until the following spring. I have had no great experience with paspalum but it seems the same. It is a secondary dormancy, the seed waiting until all conditions for germination are right. 